\title{
Negotiation and Take it or Leave it in Common Agency
}

\author{
Michael Peters \\ Department of Economics \\ University of Toronto \\ 150 St. George St. \\ Toronto, Canada \\ M5S 3G7 \\ email:peters@economics.utoronto.ca
}

First Draft -May 00

This (preliminary) draft May 7, 2000

\begin{abstract}
This short paper considers the validity of assuming that principals make their common agent a single take it or leave it contract offer instead of negotiating over the contract in a more complex way. The interest in this question arises from recent examples in the literature that illustrate equilibrium allocations that can be supported with negotiation, but not with simple take it or leave it offers. It is shown that with symmetric information, pure strategy equilibrium with take it or leave it offers are also equilibria when principals are allowed to negotiate. We also provide a class of environments in which 'pure strategy' equilibria with negotiation can all be supported with simple take it or leave it offers. The environment is restrictive, but encompasses the environment studied by Bernheim and Whinston (1986), as well as the environment involved in a simple Bertrand pricing problem similar to Klemperer and Meyer (1989).
\end{abstract}

In common agency problems, many principals simultaneously attempt to control the actions of a single agent. To accomplish this, the principals 
negotiate contracts with agents that promise the agent rewards in return for carrying out specific efforts on behalf of the principals. It has recently been observed if the negotiating scheme that a principal uses is sophisticated enough, it will allow the principal to extract information from the agent about what the other principals are doing. When this occurs, new equilibrium allocations arise that cannot be understood when principals simply offer agents take it or leave it contracts as they do in single principal problems.

Simple examples illustrating this are provided in Martimort and Stole (1997), Epstein and Peters (1997) or Peck (1995). A less abstract illustration of the idea is given with a simple reinterpretation of the argument in Parlour and Rajan (1997). In their model, multiple lenders offer a single borrower contracts that specify the principle and interest associated with a loan. The borrower (or the agent in this story) has the option of using the money in some profitable endeavour, paying the interest then keeping all the residual profit, or defaulting on the loan and retaining a fraction of the principle. When the agent's total borrowing is small, it is more profitable to repay the loan. When total borrowings are very large however, the agent prefers to default and keep a fraction of the principle of the loan.

It is tempting to model this by having each of the lenders announce a single take it or leave it principal-interest package, then letting the borrower decide which of the packages he wants to accept. However, if one of the lenders has a more complex negotiation scheme, he can improve on this outcome. The way he does this is to offer the borrower a single interest rate-principal pair, along with an option to take out a second loan at a much higher rate. The reason that the lender likes this scheme is that he knows that the agent's willingness to exercise the option on the second contract will depend on what the other lenders have offered. The borrower would not be interested in the high interest rate associated with the option unless a competitor had offered an attractive interest-principal combination to compete with the original lender. In this case the borrower will want to accept all three loans and default on each of them. The advantage to the original principal of offering this apparently unprofitable option arises from the fact that the potential competitor knows that the agent will end up defaulting if he is offered a third contract. So he is deterred from competing with the incumbent lender who enjoys a monopoly rent as a reward for his negotiation scheme. ${ }^{1}$ To put it slightly differently, the option of taking the second

\footnotetext{
${ }^{1}$ This is not exactly the argument made in Parlour and Rajan (1997) - but in essence
} 
loan gives the agent an incentive compatible way of telling the first principal that the second principal has deviated from the equilibrium path. The first principal then has the chance to punish the deviation.

The upshot is that when there are many principals, negotiation and selling schemes play a role that they do not in the single principal case. This is unfortunate for two reasons. First, the set of potential negotiation schemes is rich, involving possibly complicated extensive form games between the principal and agent. The reasoning above suggests that the principal may get some benefit from forcing the agent to play these games. Secondly the powerful results concerning common agency with symmetric information derived by Bernheim and Whinston (1986) or Dixit, Grossman, and Helpman (1997) assume that principals compete by offering agents simple take it or leave it contracts. The examples in the literature suggest that very different allocations may be supported as equilibria once principals can use more complex negotiating schemes to determine the contracts that they offer their agents.

At a conceptual level, the appropriate way to model competition among principals has been discussed in (Epstein and Peters 1997) for the general case and in (Peters 1999) more specifically for the case of common agency. The purpose of the present paper quite different. It is to try to characterize the 'cost' of ignoring negotiation and assuming that principals make simple take it or leave it contract offers. One of the principal motivations for doing this is that this is what has been done in some of the most widely cited papers in the literature on common agency (i.e. (Bernheim and Whinston 1986) and (?)). It is interesting to ask whether the results in these papers are misleading in an important way. To anticipate a little, the results here suggest that for the environments studied in these papers, the take it or leave approach is a reasonable one. In an odd way, this motivates the study of negotiation in common agency - an understanding of the general case provides some insight into cases where a naive modelling approach works reasonably well.

The principal tool used in the analysis here is a theorem in Peters (1999) that shows that no matter how complex are the negotiation mechanisms available to the principals, any equilibrium allocation that can be supported with negotiation can also be supported by allowing principals to offer agents menus of contracts that associate actions by the principal with observable

it is the same, even when there is free entry in the credit market, the potential for default will limit entry and create monopoly rents for encumbent lenders. 
efforts taken by the agent. The difference between this approach and that used by Bernheim and Whinston (1986) is simply that to reproduce all potential equilibrium allocations the principals need to be allowed to offer agents collections or menus of contracts from which they choose, rather than just a single contract. Peters (1999) also shows that any equilibrium allocation relative to this set of menus is robust in the sense that the allocation will persist as an equilibrium allocation even if the set of mechanisms available to the principals is enlarged.

It is shown first of all that in the case of symmetric information, every pure strategy equilibrium of the game in which principals offer take it or leave it contracts is also a pure strategy equilibrium if principals are allowed to offer agents menus of such contracts. ${ }^{2}$ By the robustness of equilibria relative to the set of menus established in Peters (1999), the equilibrium allocations in Bernheim and Whinston (1986) or Dixit, Grossman, and Helpman (1997) (where attention is restricted to pure strategy equilibrium) persist no matter how complex principals are allowed to make their mechanisms. Thus if principals are free to structure their negotiations as they please, one possible equilibrium outcome will be that they simply make their agents take it or leave it contract offers. Of course, as the various examples in the literature showed, alternative equilibrium allocations may also exist when more complex contracts are feasible.

To get around this, the question is then addressed whether there are interesting economics environments in which negotiation doesn not lead to new equilibrium allocations. The environment identified here is one in which there are no externalities, in the sense that the agent's ranking of the simple actions undertaken by one principal is independent of the actions taken by any other principal, and in which principals care only about their own action and the agent's effort. This is very restrictive, but fits the environment studied by Bernheim and Whinston (1986) as well as the Bertrand pricing environment studied by Klemperer and Meyer (1989) and more generally by non-linear pricing models (for example Biais, Martimort, and Rochet (1997)) where agents have quasi linear preferences. In this environment we consider a subclass of equilibria relative to the set of menus in which all of the principals and the agent use pure strategies. Within this subclass of

\footnotetext{
${ }^{2}$ Mixed strategy equilibria in take it or leave it offers among the principals will not generally survive if the principals are allowed to use menus. For examples see Epstein and Peters (1997).
} 
equilibria, it is shown that every equilibrium allocation can be supported by having principals offer only single pay for effort contracts. Examples are provided again to show that equilibrium payoffs attainable when mixed strategy equilibria (or for that matter random mechanisms) are used cannot generally be supported unless principals are allowed to use menus.

This theorem illustrates two properties of equilibria allocations attainable when principals can use complex contracts. One way menus are used is simply to exploit random behavior of the principals or the agent as a correlating device (see the examples in Peck (1995)). In equilibria where all players use pure strategies, this role for menus disappears. However this still leaves an alternative role in that menus can be used to deter deviations. This is the basic argument in Martimort and Stole (1997). A principal may not wish to change his contract offer if he believes this will affect the way the agent chooses from another principal's menu. In an environment without externalities, the agent's ranking of the options in any principal's menu is independent of what other principals are doing. So this role for menus disappears. The net result of the theorem is that if principals and agents are using pure strategies in an environment without externalities, any equilibrium allocation attainable with menus is also attainable without them. Again, using the revelation principal in Peters (1999), equilibrium allocations attainable with arbitrarily complex negotiation mechanisms in an environment without externalities, where principals and agents use pure strategies, can be supported when principals simply compete in contracts.

\section{Basic Properties}

Common agency models have the following general structure: there are $n$ principals dealing with a single agent. Each principal $j \in\{1, \ldots n\}$ controls a simple action in the set $Y_{j}$, while the agent takes some effort from a set $E$. The principal can write contracts contingent on all or part of the effort level $e \in E$ taken by the agent. To simplify it will be assumed that the sets $Y_{j}$ and $E$ are both finite. The set of feasible pay for effort contracts is denoted by $\mathcal{A}_{j}$ and this set consists of a set of feasible mappings from $E$ into the set of actions $Y_{j}$ controlled by principal $j$. Observe that the principals simple actions could be more complex than a simple monetary payment. The agent's preferences are private information and are parameterized by elements in some set $\Omega$. Principals commonly believe that the agent's preference parameter is 
distributed according to some distribution $F$ on $\Omega$.

Agents and principals have expected utility preferences. The payoff to principal $j \in\{1, \ldots, n\}$ is represented by $v_{j}: \prod_{k=1}^{n} Y_{k} \times E \times \Omega \longrightarrow[0,1]$. For the agent, payoffs are represented by the function $u: \prod_{k=1}^{n} Y_{k} \times E \times \Omega \longrightarrow$ $[0,1]$. Observe that no assumptions are made about the properties of the utility functions of either the principal or the agent.

The set $\mathcal{A}_{j}$ of feasible contracts varies in different applications depending on how sensitive the principal's contract can be to the agent's action. Let $\mathcal{E}_{j}$ be a partition of $E$ that captures principal $j$ 's ability to contract on the agent's effort in the sense that every feasible contract for principal $j$ must be measurable with respect to (the $\sigma$ field generated by) the partition $\mathcal{E}_{j}$. For example, in Bernheim and Whinston (1986) principals are interpreted as lobbyists making transfers to a government agency in an attempt to influence policy (the agent's action). Transfers can depend on the policy that is chosen, so in this sense each principal can write contracts fully contingent on the agent's effort. Then $\mathcal{E}_{j}$ is just the set consisting of all single element subsets of E. In Martimort and Stole (1997) or Biais, Martimort, and Rochet (1997) the principals are sellers while the agent is a buyer. The principals are allowed to write contracts in which the prices they charge depend on the quantity that the agent buys from them (but not on the quantity the agent buys from other principals). Then think of $E \subset Q^{n}$ where $Q$ is some finite set of feasible demands. Then $\mathcal{E}_{j}$ is the collection of sets of the form

$$
B(x)=\left\{\left(q_{1}, \ldots q_{n}\right): q_{j}=x ; \forall i q_{i} \in Q\right\} .
$$

Of course, it is possible that the principal's contract might have to be completely independent of the agent's action. In this case $\mathcal{E}_{j}=E$.

Principals decide which contracts to offer by designing a mechanism. A mechanism specifies the rules that guide communication between the principal and agent. The basic idea is that the agent sends messages to the principal. Based on these messages, the principal takes an action and responds to the agent. Finally the agent chooses the effort level he wants.

For any measurable set $X$, Let $\triangle(X)$ denote the set of probability measures on $X$. When a topology on $\triangle(X)$ is needed, the topology of weak convergence is assumed. For any $x \in \triangle(X)$, let supp $x$ be the support of $x$. Let $C_{j}$ be the (measurable) set of messages that the agent can send to a principal $j$. Let $\mathcal{R}_{j}$ be the (measurable) set of responses that principals can send back to the agent. These message spaces are intended to be quite 
general in the degree and nature of the communication about the other principals' mechanisms that they permit. For example, the messages could allow the agent to communicate the mechanisms being used by other principals.

The role of the principal's messages is to provide the agent with information about the action that the principal has chosen. An indirect mechanism $\gamma_{j}$ for principal $j$ specifies a measurable map from $C_{j}$ into $\triangle\left(\mathcal{A}_{j} \times \mathcal{R}_{j}\right)$. For each message the agent sends, the principal responds by committing himself to a joint probability measure over actions and messages. To simplify the notation a little, it will be assumed that the set of feasible actions $\mathcal{A}_{j}$ is the same for each principal. Then define $\Gamma$ to be a topological space of feasible indirect mechanisms available to each of the principals. Let $\gamma=\left\{\gamma_{1} \ldots \gamma_{n}\right\} \in \Gamma^{n}$ refer to the entire array of mechanisms offered by the principals. Without loss of generality, it can be assumed that the message spaces $C_{j}$ and $\mathcal{R}_{j}$ are the same for each principal ${ }^{3}$. Then each mechanism $\gamma_{j}$ is a measurable map from $C$ into $\triangle(\mathcal{A} \times \mathcal{R})$.

Agent behavior in each mechanism depends on the agent's valuation and on the mechanisms that he or she observes being offered by the other principals. A communications strategy is a measurable mapping $\tilde{c}: \Omega \times \Gamma^{n} \rightarrow$ $\triangle\left(C^{n}\right)$ that describes the (probability distribution over) messages that the agent will send to the principals as a function of the agent's type and the array of mechanisms that he is offered by the principals. A decision strategy $\tilde{\pi}: \Omega \times \Gamma^{n} \times C^{n} \times R^{n} \rightarrow \triangle(E)$ is a measurable mapping that describes the probability distribution the agent will use to choose his action as a function of his type, the array of mechanisms that he has been offered, the messages he has sent, and the array of messages received from the principals. The pair $(\tilde{c}, \tilde{\pi})$ together constitute a continuation strategy for the agent. Say that the continuation strategy $(\widetilde{c}, \tilde{\pi})$ is a continuation equilibrium if for every array of mechanisms $\gamma \in \Gamma^{n}$ offered by the principals and for almost every (with respect to $F)$ valuation $\omega \in \Omega,($ i) the randomizations $\tilde{c}(\omega, \gamma)$ and $\tilde{\pi}(\omega, \gamma, \cdot, \cdot)$ maximize

$$
\int \ldots \int\left\{\int u\left(a_{1}(e), \ldots a_{n}(e), e, \omega\right) d \pi\left(c_{1}, \ldots c_{n}, r_{1}, \ldots r_{n}\right)\right\} d \gamma_{1}\left(c_{1}\right) \ldots d \gamma_{n}\left(c_{2}\right) \text {. }
$$

A continuation equilibrium $(\tilde{c}, \tilde{\pi})$ determines a normal form game among the

\footnotetext{
${ }^{3}$ If the message spaces are different, simply redefine the message spaces used by each firm to be the union of the messages spaces being used by all firms, then redefine principal $j$ 's allocation rule $\gamma_{j}$ so that it produces an undesirable action whenever the agent sends a message that is not in the message space that the principal wants.
} 
principals in which the actions spaces are $\Gamma$ for each principal and payoffs are given by

$$
\begin{gathered}
\bar{v}_{j}\left(\gamma_{1}, \ldots \gamma_{n}, \tilde{c}, \tilde{\pi}\right)= \\
\int \cdots \int\left\{\int v(a(e), e, \omega) d \tilde{\pi}\left(\omega, \gamma_{1}, \ldots \gamma_{n}, c, r\right)\right\} d \tilde{c}\left(\gamma_{1}, \ldots \gamma_{n}\right) \cdot d \gamma_{1} \cdots d \gamma_{n}
\end{gathered}
$$

An equilibrium relative to the set of feasible mechanisms $\Gamma$ is an array of randomizations $\left\{\delta_{1}, \ldots \delta_{n}\right\}$ and a continuation equilibrium $(\tilde{c}, \tilde{\pi})$ such that $\left\{\delta_{1}, \ldots \delta_{n}\right\}$ is a Nash Equilibrium for the normal form game defined by the continuation equilibrium $(\tilde{c}, \tilde{\pi})$.

Let $m$ be a probability measure on the set $Y \times \triangle(Y)$. Let $m_{y}$ be the marginal measure on $Y, m_{\lambda}$ the marginal measure on $\triangle\left(Y_{j}\right)$ and $m(\cdot \mid \lambda)$ the conditional measure. The probability measure $m$ is said to be consistent if for each $m \in \Phi_{j} ; \lambda(\cdot)=m(\cdot \mid \lambda)$ for all $\lambda$ in the support of $m_{\lambda}$. Let $\Phi$ be any subset $\triangle(Y \times \triangle(Y))$. The set $\Phi$ is said to be consistent if each of the measure in $\Phi$ is consistent. Consistent probability measures randomly choose a simple action from $Y$ and a message consisting of a probability measure over $Y$. Consistency means that the message accurately conveys the true (conditional) probability measure over actions. One property of consistent measures that is relevant for common agency is the fact that

$$
\int \lambda(\cdot) d m_{\lambda}=\int m(\cdot \mid \lambda) d m_{\lambda}=m_{y}
$$

by the definition of conditional probability. In other words, the marginal distribution on $Y_{j}$ coincides with the 'reduced' distribution on $Y_{j}$ associated with the marginal $m_{\lambda}$. In the sequel, the principal will choose a simple action from $Y$ then send a message to the agent that is correlated (possibly perfectly) with the action choice. If the action that the principal chooses is random, then the message that the agent receives is also random. If the randomizing device used to select actions and messages is consistent then the ex ante distribution of actions faced by the agent will coincide with the expected message.

There are some notable examples of consistent sets of measure. One special (and relatively simple) class contains only measures that assign unit probability mass to a single point in $Y$ and to a single measure in $\triangle(Y)$ that in turn assigns unit mass to the same point. 'Random' devices of this kind 
simply choose and element of $Y_{j}$ non-randomly, then inform the agent of this choice. ${ }^{4}$ Another interesting set is the set of joint distributions $\Phi$ such that the projection of the support of each $\phi \in \Phi$ onto $\triangle(Y)$ is contained in the set of degenerate distributions that assign unit mass to a single point in $Y$. These distributions choose elements of $Y$ randomly, but then send messages that reveal the choice exactly.

Let $\mathcal{A}^{*}$ be the set of joint probability measures a over $\mathcal{A} \times \triangle(\mathcal{A})$. Any such measure induces a family of joint probability measures on $Y \times \triangle(Y)$ defined by

$$
m_{e}^{a}(B)=a\{x \in \mathcal{A} \times \triangle(\mathcal{A}): x(e) \in B\}
$$

for each $B \subset Y \times \triangle(Y)$. To continue, an obvious extension is to say that $a \in \mathcal{A}^{*}$ is consistent if every member of the family of measures $m_{e}^{a}$ is consistent, and that any collection of measures $A \in \mathcal{A}^{*}$ is consistent if each of its members is consistent. A menu is a mechanism $\gamma_{j}$ whose message spaces are given by $C=\mathcal{A}^{*}$ and $\mathcal{R}=\triangle(\mathcal{A})$, and whose range $\gamma_{j}(\cdot)$ is a closed and consistent subset of $\mathcal{A}^{*}$. In other words, a menu is simply a closed subset of $\mathcal{A}^{*}$ containing consistent probability measures. By sending a message to the principal the agent essentially chooses a consistent probability measure. This probability measure chooses a contract and sends a message to the agent describing a probability measure over contracts. The agent uses this to associate a measure over simple actions with each level of effort. The notation $\Gamma^{*}$ is used to refer to the set of menus.

The following Theorem is proved in Peters (1999):

Theorem 1 Let $\left\{v_{1}^{*}(\omega), \ldots v_{n}^{*}(\omega), u^{*}(\omega)\right\}$ be the equilibrium payoffs of each of the principals and the agent when the agent's type is $\omega$ and the set of feasible mechanisms is $\Gamma$. Then there is an equilibrium relative to the set of menus $\Gamma^{*}$ that preserves these payoffs for all $\omega$ in the support of $F$.

The theorem says that all payoffs that can be supported as equilibrium payoffs with competition among the principals can be supported when the principals are restricted to use menus. Furthermore, Peters (1999) also

\footnotetext{
${ }^{4}$ In this simple case with degenerate probability measures consistency requires that the message coincide with the simple action chosen. So an inconsistent measure would simply misinform the agent about the action. If the agent knows the joint distribution that the principal has chosen, he will ignore the message since he knows the action the principal has taken anyway. That is why inconsistent random devices need not be considered.
} 
shows that equilibrium payoffs supported with menus are all 'interesting' in the sense that these equilibrium payoffs persist when principals are allowed to use more complex mechanisms (provided that the continuation equilibrium in the enlarged set of mechanisms is constructed properly). This suggest that menus provide a powerful simplification of a complicated conceptual problem. As mentioned above, the menus that the principals need to offer are menus of 'contracts', not menus of simple actions. In the problem discussed Bernheim and Whinston (1986) for example, each principal should offer the agent the choice between a number of different pay for effort schemes. In a simple problem with Bertrand competition, each seller should offer the buyer a menu of non-linear pricing schemes.

The set of menus is conceptually simple relative to the set of mechanisms. Apart from the examples in the literature, this may lead to interesting applications. ${ }^{5}$ The primary focus in this paper, however, is to try to use the theorem above to determine the usefulness of the standard approach. In Bernheim and Whinston (1986), the 'standard approach' ignores the possibility that principals might offer menus and instead has them competing by offering single elements from $\mathcal{A}^{*}$ (the set of joint probability measures on contracts and messages). So in the remainder of the paper, an attempt is made to contrast equilibrium outcomes when principals offer the agents menus (i.e. collections of contracts from which the agent can choose) to outcomes when each principal is allowed to offer the agent only a single contract. Let $\Gamma_{C}$ denote the set of contracts (i.e., probability measures on $\mathcal{A} \times \triangle(\mathcal{A})$ ). Note that $\Gamma_{C} \subset \Gamma^{*}$ (in the sense that single element menus are nonetheless menus). Formally a menu is a map $\gamma: \mathcal{A}^{*} \rightarrow \mathcal{A}^{*}$. To make the argument slightly more transparent we will sometimes speak about choosing the member element $g$ from the menu $\gamma$. Formally this means sending a message $g \in \mathcal{A}^{*}$ to the principal such that $\gamma(g)=g$.

\section{Pure Strategy Theorem}

The first theorem applies directly to the problem studied by Bernheim and Whinston (1986) or Dixit, Grossman, and Helpman (1997). The principals and the agent are said to have symmetric information if the distribution $F$ of types for the agent is degenerate.

\footnotetext{
${ }^{5}$ For example, the application described by Parlour and Rajan (1997).
} 
Theorem 2 Suppose the principals and the agent have symmetric information and let $\gamma^{*}=\left\{\gamma_{1}^{*}, \ldots \gamma_{n}^{*}\right\}$ be any pure strategy equilibrium relative to the set of contracts $\Gamma_{C}$. Then $\gamma^{*}$ is also an equilibrium relative to the set of menus $\Gamma^{*}$.

Proof. Let $(\pi, c)$ be the continuation equilibrium relative to $\Gamma_{C}$. Since each $\gamma_{j}^{*} \in \Gamma_{C}$ it follows that $\gamma_{j}^{*}$ contains a single element $g_{j}^{*} \in \mathcal{A}^{*}$. Then principal $j$ 's payoff could be written as

$$
\int \cdots \int\left\{\int v\left(a_{1}(e), \ldots a_{n}(e), e\right) d \pi\left(\gamma_{1}^{*}, \ldots \gamma_{n}^{*}, r_{1}, \ldots r_{n}\right)\right\} d g_{1}^{*} \ldots d g_{n}^{*}
$$

The problem at hand is simply to extend the continuation equilibrium from $\Gamma_{C}$ to all of $\Gamma^{*}$ in a way that preserves the equilibrium allocation. For any array of offers $\left\{\gamma_{j}^{\prime}\right\}$ such that $\gamma_{j}^{\prime} \in \Gamma_{C}$ for all $j$ define

$$
\tilde{\pi}\left(\gamma_{1}^{\prime}, \ldots \gamma_{n}^{\prime}, r_{1}, \ldots r_{n}\right)=\pi\left(\gamma_{1}^{\prime}, \ldots \gamma_{n}^{\prime}, r_{1}, \ldots r_{n}\right)
$$

which simply means that in the continuation equilibrium relative to $\Gamma^{*}$, the agent responds to single element menus (contracts) the same way that he does relative to $\Gamma_{C}$. If two or more offers in the array $\left\{\gamma_{j}^{\prime}\right\}$ are different from the corresponding offer $\gamma^{*}$, the continuation strategy for the agent can be defined in any convenient way ${ }^{6}$. If a single principal $j$ deviates from $\gamma_{j}^{*}$ to a non-degenerate menu $\gamma^{\prime}$ of contracts in $\Gamma^{*}$ choose $\bar{g} \in \gamma^{\prime}$ and $\bar{\pi}^{\prime}$ to maximize

$$
\int \ldots \int\left\{\int u\left(a_{1}(e), \ldots a_{n}(e), e\right) d \pi\left(r_{1}, \ldots r_{n}\right)\right\} d g_{1}^{*} \cdots d g_{j-1}^{*} d g^{\prime} \cdots d g_{n}^{*}
$$

Now define

$$
\tilde{\pi}\left(\gamma_{1}^{*}, \cdots \gamma_{j-1}^{*}, \gamma^{\prime} \ldots \gamma_{n}^{*}, r_{1}, \ldots r_{n}\right)=\bar{\pi}\left(r_{1}, \ldots r_{n}\right)
$$

and

$$
\tilde{c}_{j}\left(\gamma_{1}^{*}, \cdots \gamma_{j-1}^{*}, \gamma^{\prime} \ldots \gamma_{n}^{*}\right)=\bar{g}
$$

In words, when principal $j$ offers the menu $\gamma^{\prime}$, the agent selects $\bar{g}$ from the menu and then acts as if the principal had offered a degenerate menu consisting only of the offer $\bar{g}$. The strategy $\pi\left(\gamma_{1}^{*}, \cdots \gamma_{j-1}^{*}, \bar{\gamma} \ldots \gamma_{n}^{*}, r_{1}, \ldots r_{n}\right)$

\footnotetext{
${ }^{6}$ An optimal continuation strategy exists because expected utility implies that preferences are linear (hence continuous) in the distributions in $\Gamma^{*}=\triangle(\mathcal{A} \times \triangle(\mathcal{A}))$, while by definition, menus are compact (in the weak topology) subsets of $\Gamma^{*}$.
} 
must be at least as good as $\bar{\pi}$, else the definition of $\bar{\pi}$ as the maximizer is contradicted.

The construction ensures that when principal $j$ offers the non-degenerate menu $\gamma^{\prime}$ the agent responds in a way that gives the same principal the same payoff he would have received if he had deviated and offered the degenerate menu consisting only of the contract $\bar{g}$. Since $\gamma_{j}^{*}$ is a best reply to $\gamma_{1}^{*}, \cdots \gamma_{n}^{*}$ by the definition of equilibrium, this deviation cannot be profitable.

By the robustness theorem, since the contracts $\gamma^{*}$ constitute an equilibrium relative to the set of menus, then the allocation associated with these contracts can be supported as an equilibrium allocation no matter what the set of feasible mechanisms is for the principals. In one sense this justifies the approach adopted in Bernheim and Whinston (1986) and Dixit, Grossman, and Helpman (1997). Yet most of the examples of the use of menus cited in the literature refer to equilibrium allocations that can only be supported when principals are allowed to offer menus. The question remains whether there are interesting environments where all equilibrium allocations can be determined by allowing principals to compete in contracts alone.

\section{Environments without Externalities}

The literature has identified two distinct sorts of equilibria with menus that are eliminated by restricting contracts. In one sort of equilibrium deviations by one principal are deterred by the possibility that the agent will change his selection from the other principal's menu. This means that principals indirectly respond to one another's deviations by modifying their own actions (an example is Martimort and Stole (1997)). This is the sense in which complicated communications mechanisms allow the agent to communicate market information to the principal (Epstein and Peters (1997)). Alternatively, (Peck (1995)) the agent can act as a correlating device for the principals (or the principals can act as correlating devices for the agent) in a way that generates payoffs that cannot be attained in the absence of such devices.

To illustrate the latter role consider the following trivial example - two principals play a coordination game in which each of them has two possible simple actions. If both principals take action $\mathrm{A}$, each of them gets a payoff 5 , while if they coordinate on action $B$ each of them gets a payoff 1 . Each principal gets 0 if they fail to coordinate. There is an agent who takes no effort, and who gets payoff 0 no matter what happens to the principals. 
There is an equilibrium in menus in which each principal offers the agent the choice between action $A$ and $B$. The agent choose $A$ from both principals half the time and $B$ from both principals the other half of the time. If one principal deviates and eliminates an option from the menu, the agent is expected to choose the combination of actions that gives the principals 0 payoff. The payoff to the principals in this equilibrium is 3 , and this cannot be supported if principals are forced to choose a single action.

It is hard to see how this role for menus could be ruled out by imposing interesting restrictions on the environment alone. The necessary restrictions would have to rule out mixing by the agent. However, if mixing can be ruled out for some other reason, it may be reasonable to focus contracts alone without worrying about menus. This brings up the much more difficult problem of agent indifference. In general, menus may consist of various alternatives among which the agent is indifferent. Principals, on the other hand, may not be indifferent about how the agent chooses from the menu. If this is the case, deviations can be used to trigger changes in the way the agent chooses from the menu, and this can be used to support all kinds of equilibrium outcomes that are unattainable without menus.

As an extreme example of this, consider the following

$$
\begin{array}{ccc} 
& a & b \\
a & 0,0,0 & 2,1,0 \\
b & 1,2,0 & 3,3,0
\end{array}
$$

In this each principal has two possible simple actions $a$ and $b$. The agent takes no effort and is completely indifferent among the combinations of simple actions taken by the principals. For simplicity, rule out random contracts. Then the set of feasible contracts is simply the set of simple actions available to the principal. If we model the game as one where principals compete in simple contracts, the only pure strategy equilibrium is the one where each principal chooses the simple action $b$. However, the outcomes $(a, b)$ and $(b, a)$ can be supported in competition in menus by having each principal offer the agent the menu consisting of both outcomes $a$ and $b$. If either principal deviates, the agent selects the action from the non-deviating principal's menu that gives the deviator the lowest payoff. It is reasonably easy to see from this example, that if the agent is wholly indifferent about the principals' simple actions, then any outcome that gives both principals a payoff at least as large as their maximin payoff can be supported with menus. 
There are, nonetheless, some interesting environments in which competition in contracts is sufficient to understand most equilibrium behavior. The qualifier in the previous sentence is related to the fact, as already established, that continuation equilibria involving mixing cannot ordinarily be supported by having sellers compete in contracts alone.

Say that the no-externalities assumption holds relative to some set of measures $\Phi \subset \triangle\left(Y_{j} \times \triangle\left(Y_{j}\right)\right)$ if the agent has a strict preference ordering over the elements of $\Phi$ that is independent of his effort and the randomizations in $\Phi$ being offered by the other principals, and the principal's utility is determined only by his own randomization and the agent's effort. Formally for each $j$

(i) for each pair $\left(y_{1}, \ldots, y_{j-1}, y_{j+1}, \ldots y_{n}\right)$ and $\left(y_{1}^{\prime}, \ldots, y_{j-1}^{\prime}, y_{j+1}^{\prime}, \ldots y_{n}^{\prime}\right)$ and for each effort level $e \in E$;

$$
v\left(y_{1}, \ldots y_{j}, \ldots y_{n}, e, \omega\right)=v\left(y_{1}^{\prime}, \ldots y_{j}, \ldots y_{n}^{\prime}, e, \omega\right)
$$

and

(ii) if there is an $e \in E$ and $\phi_{-j}$ such that

$$
\begin{gathered}
\int \cdots \int U\left(y, y_{-j}, e, \omega\right) d \phi(y) d \phi_{-j}\left(y_{-j}\right)> \\
\int \cdots \int U\left(y, y_{-j}, e, \omega\right) d \phi^{\prime}(y) d \phi_{-j}\left(y_{-j}\right)
\end{gathered}
$$

then for every $e^{\prime} \in E$ either

$$
\begin{gathered}
\int \cdots \int U\left(y, y_{-j}, e^{\prime}, \omega\right) d \phi(y) d \phi_{-j}\left(y_{-j}\right)> \\
\int \cdots \int U\left(y, y_{-j}, e^{\prime}, \omega\right) d \phi^{\prime}(y) d \phi_{-j}\left(y_{-j}\right)
\end{gathered}
$$

for all $\phi_{-j} \in \Phi^{N-1}$ or

$$
\begin{gathered}
\int \cdots \int U\left(y, y_{-j}, e^{\prime}, \omega\right) d \phi(y) d \phi_{-j}\left(y_{-j}\right)= \\
\int \cdots \int U\left(y, y_{-j}, e^{\prime}, \omega\right) d \phi^{\prime}(y) d \phi_{-j}\left(y_{-j}\right)
\end{gathered}
$$

and $\int v\left(y_{j}, e, w\right) d \phi\left(y_{j}\right)=\int v\left(y_{j}, e, w\right) d \phi^{\prime}\left(y_{j}\right)$ for all $\phi_{-j} \in \Phi^{N-1}$. 
The complexity of the second condition stems from the fact that in many problems the agent can take an action that insulates him completely from the action of the principal - for example, the agent can refuse to trade with the principal. If so, then neither the agent or the principal involved should care what action the principal tries to implement.

The no-externalities assumption is obviously a strong one, but there are a couple of interesting environments that satisfy it provided the set of measures is suitably restricted. The simplest is probably the model in which several sellers compete in price to sell to a single buyer. Since sellers can condition price on the quantity that the agent chooses to purchase, this might be modelled as an equilibrium in supply functions Klemperer and Meyer (1989). In this case let $\Phi$ be the set of degenerate distributions that assign all probability mass to a single price and to a message that assigns all probability mass to that same price. Then $\Phi$ is effectively the set of possible price offers. The buyer will strictly prefer the lowest price offered unless he or she chooses not to buy from the seller. In that case, the buyer will be indifferent about the seller's price. Each seller's utility is just his own revenue, which is the product of the per unit price that he offers and the quantity that the buyer purchases (which would be the agent's effort in this interpretation). Thus the no-externalities assumption is satisfied relative to the set of prices. Notice the environment will not typically satisfy the no-externalities assumption for alternative sets of distributions $\Phi$. To see why, suppose that distributions are admitted into $\Phi$ in which the marginal measure $m_{y}$ is non-degenerate (or the seller offers the buyer a lottery over price). Suppose as well that some seller has offered the buyer a choice between a fixed price and a lottery over price (but that the message that the seller sends with the random contract perfectly informs the buyer about price). Suppose that the two contracts are such that if the buyer purchases all his output from the seller, then the buyer is just indifferent between the two contracts. Then when the other seller sets a very high price, the buyer will (weakly) prefer the non-random price. On the other hand, if the other seller matches the non-random price of the first seller, then the buyer will strictly prefer the random contract because of the option value that it provides (i.e., to buy from the first seller when his random device generates a lower price). So the non-externalities assumption fails.

An alternative environment without externalities is the one studied by Bernheim and Whinston (1986) in which multiple principals offer the agent a (non-random) payment in return for some effort that the agent undertakes. 
The simple actions available to each principal are just payments, and if random payment schemes are ruled out, then the agent will always strictly prefer the largest payment. Principals' utility depends on the utility that they get from the agent's effort, and on the payment that they make, so again the environment is separable.

An example was described above in which randomization by the agent along with menus could be used to generate correlated equilibrium allocations in contracts. A very similar effect can be accomplished if principals are allowed to use random mechanisms that involve randomized messages sent to the agent. Again, the best way to illustrate this is probably a trivial example that extends the coordination example given above.

$\begin{array}{rrcl} & a & b & c \\ a & 5,5,0 & 0,0,0 & 0,0,0 \\ b & 0,0,0 & 1,1,0 & 0,0,0 \\ c & 0,0,0 & 0,0,0 & 0,0,0\end{array}$

As before, the first two payoffs in each cell are the payoffs to the two principals, while the third payoff is the agent's payoff (which is always 0). One equilibrium in menus has each principal randomizing initially over menus, selecting the menus $\{a, b\}$ and $\{a, b, c\}$ with equal probability then sending the agent a message saying which choice he made. If both principals send the same message, the agent chooses $a$ from both menus while if the messages differ, the agent chooses $b$. As before, any deviation from this behavior by the principals induces the agent to punish by selecting the elements from the non-deviator's menu that is worst for the deviator.

The agent plays a pure strategy in this example, but the payoff to the principals is again the correlated equilibrium payoff 2 , which is not supported if the principals compete in contracts alone. This assumption doesn't satisfy the no-externalities assumption, ${ }^{7}$ but similar arguments can be made with more complicated examples in a no-externalities environment. The point of the example is just to illustrate that to prove some kind of revelation principal when principals compete in contracts alone requires restrictions on the set of feasible contracts to rule out randomization, for the same reason that it is necessary to rule out randomization by the agent. In what follows we simply rule out mechanisms in which the principal can communicate with

\footnotetext{
${ }^{7}$ It does fit for the agent, but the principals' payoffs depend on what the other principals do which is necessary in a coordination game.
} 
the agent before the agent takes effort (Martimort and Stole (1997) uses this assumption for example). The revelation principle 1 is readily adjusted to fit this situation - menus are simply mechanisms in which the set of feasible messages for the agent is $\triangle(\mathcal{A})$ while the set of feasible messages for the principal is $\mathcal{R}=\emptyset$. Rather than pursuing this formally, we simply impose a restriction on the set of feasible menus. Let $\mathcal{A}^{0} \subset \mathcal{A}^{*}$ be the set of feasible probability measures that principals can offer. Each contract in $a \in \mathcal{A}^{0}$ induces a family of probability measures $m_{e}^{a}$ as in 1 above. Let $\Phi^{0} \equiv \cup_{a \in \mathcal{A}^{0}, e \in E} m_{e}^{a}$. By the revelation principle 1, the set of measures $\Phi^{0}$ is consistent. The set of indirect mechanisms satisfies no communication by the principal if every $m \in \Phi^{0}$ has the property that $m_{\triangle}$ is a degenerate measure with all of its mass assigned to the distribution $m_{y}$. In words, this means that the principal is constrained always to send the same message to the principal. Consistency requires that this message be the marginal distribution on the set of simple actions.

Theorem 3 Suppose the set of feasible mechanisms satisfies no communication by the principal and that the environment satisfies the no-externalities assumption relative to $\Phi^{0}$. Let $\left\{\gamma_{1}^{*}, \ldots \gamma_{n}^{*}\right\}$ be a pure strategy equilibrium relative to the set of menus $\Gamma^{*}$ and suppose that in the continuation equilibrium associated with $\Gamma^{*}$ the agent uses pure strategies. Then there exist an equilibrium $\left\{\tilde{\gamma}_{1}, \ldots \tilde{\gamma}_{n}\right\}$ relative to the set of contracts $\Gamma_{C}$ which preserves the payoffs of the principals and the agent, for almost all values of the agent's type.

Proof. Let $\gamma$ be an arbitrary array of menus. For each $e \in E$ and for every $j=1, \ldots n$ there is a $g_{j}^{e} \in \gamma_{j}$ such that

$$
\begin{gathered}
U\left(y_{j}, y_{-j}, e, \omega\right) d g_{1}(e) \cdots d g_{j}^{e}(e) \cdots d g_{n}(e) \geq \\
U\left(y_{j}, y_{-j}, e, \omega\right) d g_{1}(e) \cdots d g^{\prime}(e) \cdots d g_{n}(e)
\end{gathered}
$$

for each $g^{\prime} \in \gamma_{j}$ such that $g^{\prime} \neq g_{j}^{e}$; for any $g_{k} \in \gamma_{k}$ for each $k \neq i$; and for every $\omega \in \Omega$. This inequality will be strict unless the agent of type $\omega$ is indifferent to all contracts in $\gamma_{j}$ when he takes effort $e$. All this follows from the separability of the agent's preferences. By the definition of $\mathcal{E}_{j}$, for each $B \in \mathcal{E}_{j}, g_{j}^{e}(e)=g_{j}^{e^{\prime}}\left(e^{\prime}\right)$ for each pair $e, e^{\prime} \in B$ since every $g^{\prime} \in \gamma_{j}$ must 
be measurable with respect to $\mathcal{E}_{j}$. As $E$ and $Y_{j}$ are both finite, the random function $g_{\gamma_{j}}^{0}: E \rightarrow \triangle(Y)$ defined by

$$
g_{\gamma_{j}}^{0}(e)=g_{j}^{e}(e)
$$

is also an element of $\triangle(\mathcal{A})$ whose support consists of functions from $E \rightarrow Y$ measurable with respect to $\mathcal{E}_{j}$.

Again consider an arbitrary array of menus $\gamma$. Now replace each of these menus with the degenerate menu consisting of the single element $g_{\gamma_{j}}^{0}(\cdot)$. Let $e^{*}(\omega)$ be the equilibrium effort level chosen by the agent of type $\omega$ when principals offer the contracts $\gamma$. This function exists by the assumption that the agent uses a pure strategy in the continuation equilibrium relative to $\Gamma^{*}$. Observe that $e^{*}(\omega)$ must describe an optimal effort level for every agent type when the principals offer degenerate menus $g^{0}$. For if this were false, there would be an effort level and a selection of contracts that the agent could have made relative to $\gamma$ that would have improved his payoff for some value of his type, a contradiction of the assumption that $e^{*}(\omega)$ describes equilibrium effort. ${ }^{8}$ By separability, an agent of type $\omega$ must either select the contract $g_{j}^{e^{*}(\omega)}$ from principal $j$ in equilibrium, or be indifferent to all of the contracts that principal $j$ offers. In either case, the payoff that the principal and agent get from the combination of the effort level $e^{*}(\omega)$ and the randomization $g_{j}^{e^{*}(\omega)}$ is the same as their payoff when the principals offer $\gamma$. Taking expectations over the agent's possible types then guarantees that each principal's expected payoff when each of them offers $g_{\gamma_{j}}^{0}$ is the same as it would be if each of them offers $\gamma_{j}$.

Let $\bar{V}_{j}(\gamma)$ and $\bar{U}(\gamma, \omega)$ be principal $j$ 's and agent $\omega$ 's payoff when the array of menus is $\gamma$. This argument says that for any arbitrary array of menus $\gamma, \bar{V}_{j}(\gamma)=\bar{V}_{j}\left(g_{\gamma}^{0}\right)$ and $\bar{U}(\gamma, \omega)=\bar{U}\left(g_{\gamma}^{0}, \omega\right)$ for each $j$ and $\omega \in \Omega$. Now consider the equilibrium array of menus $\gamma^{*}$ and the associated set of simple contracts $g_{\gamma^{*}}^{0}$. Consider any unilateral deviation $\gamma_{j}^{\prime}$ from the array $g_{\gamma^{*}}^{0}$. Then from this argument

$$
\bar{V}\left(\gamma_{j}^{\prime}, \gamma_{-j}^{*}\right)=\bar{V}\left(g_{\gamma_{j}^{\prime}}^{0}, g_{\gamma_{-j}^{*}}^{0}\right)=\bar{V}\left(\gamma_{j}^{\prime}, g_{\gamma_{-j}^{*}}^{0}\right) \leq \bar{V}\left(\gamma_{j}^{*}, \gamma_{-j}^{*}\right)=\bar{V}\left(g_{\gamma_{j}^{*}}^{0}, g_{\gamma_{-j}^{*}}^{0}\right)
$$

\footnotetext{
${ }^{8}$ Observe that this part of the argument does not require separability. It would be true without separability provided that the agent uses a pure strategy in the continuation. If the agent mixes among contracts and efforts, some of the choices that he made in the original continuation would no longer be available to him after the principals replaced their menus with single contracts.
} 
The first two inequalities follow because the argument above is true for any array of contracts. The inequality follows from the fact that $\gamma^{*}$ is an equilibrium in menus. Thus the array of contracts $g_{\gamma^{*}}^{0}$ not only preserves all players payoffs, it is also an equilibrium relative to the set of menus $\Gamma^{*}$.

The upshot is that if there is some interesting allocation that can be discovered by analyzing (pure strategy) equilibrium in which principals use complex negotiation schemes to determine their contracts, then the allocation can also be characterized and studied by having principals compete by offering the agent take it or leave it contracts exactly as is done the standard references like Bernheim and Whinston (1986) or Dixit, Grossman, and Helpman (1997).

\section{Conclusion}

Complex negotiations can result interesting new behavior in common agency when there are many principals. Standard approaches to common agency developed by Bernheim and Whinston (1986) or Dixit, Grossman, and Helpman (1997) ignore this. This paper shows that provided one is interested in pure strategy equilibria, these approaches lead to errors in omission rather than errors in commission. With symmetric information, pure strategy equilibria in take it or leave it contracts can be supported as equilibria when principals are allowed to use arbitrarily complicated negotiation schemes. Furthermore pure strategy equilibria involving negotiation schemes that generate nonrandom outcomes can be supported as equilibria when principals compete in take it or leave it contracts.

It may be possible to extend the theorem above to allow for random outcomes and mixed strategy equilibria if one is interested in correlated equilibria of games in which principals offer take it or leave it contracts. Since the environment without externalities is so restrictive, it would seem more useful to accept menus of contracts as the appropriate way for principals to compete in order to analyze the impact of externalities more directly.

\section{References}

Bernheim, B. D., and M. D. Whinston (1986): "Common Agency," Econometrica, 54(4), 923-942. 
Biais, B., D. Martimort, And J.-C. Rochet (1997): "Competing Mechanisms in a Common Value Environment," Discussion paper, Universite de Toulouse.

Dixit, A., G. Grossman, and E. Helpman (1997): "Common agency and coordination: General theory and application to government policymaking," pp. 752-769.

Epstein, L., And M. Peters (1997): "A Revelation Principle for Competing Mechanisms," University of Toronto.

Klemperer, P. D., And M. A. Meyer (1989): "Supply Function Equilibria in Oligopoly under Uncertainty," Econometrica, 57(6), 1243-77.

Martimort, D., and L. Stole (1997): "Communications Spaces, Equilibria Sets and the Revelation Principle under Common Agency," University of Chicago unublished manuscript.

Parlour, C. A., and U. Rajan (1997): "Competition in Loan Contracts," Discussion paper, Carnegie Mellon University.

PECK, J. (1995): "Competing Mechanisms and the Revelation Principle," Ohio State University Manuscript, p. 12.

Peters, M. (1999): "Common Agency and the Revelation Principle," Discussion paper, University of Toronto. 